\title{
WASP-8b: a retrograde transiting planet in a multiple system ${ }^{\star}, \star \star$
}

\author{
D. Queloz ${ }^{1}$, D. Anderson², A. Collier Cameron ${ }^{3}$, M. Gillon ${ }^{4}$, L. Hebb ${ }^{3}$, C. Hellier², P. Maxted ${ }^{2}$, F. Pepe ${ }^{1}$, D. Pollacco ${ }^{5}$, \\ D. Ségransan ${ }^{1}$, B. Smalley ${ }^{2}$, A. H. M. J. Triaud ${ }^{1}$, S. Udry ${ }^{1}$, and R. West ${ }^{6}$ \\ 1 Observatoire Astronomique de l'Université de Genève, Chemin des Maillettes 51, 1290 Sauverny, Switzerland \\ e-mail: Didier.Queloz@unige.ch \\ 2 Astrophysics Group, Keele University, Staffordshire, ST55BG, UK \\ 3 School of Physics \& Astronomy, University of St Andrews, North Haugh, KY16 9SS, St Andrews, Fife, Scotland, UK \\ 4 Institut d'Astrophysique et de Géophysique, Université de Liège, Allée du 6 Août, 17, Bat. B5C, Liège 1, Belgium \\ 5 Astrophysics Research Centre, School of Mathematics \& Physics, Queens University, University Road, Belfast, BT71NN, UK \\ ${ }^{6}$ Department of Physics and Astronomy, University of Leicester, Leicester, LE17RH, UK
}

Received 12 April 2010 / Accepted 21 June 2010

\section{ABSTRACT}

\begin{abstract}
We report the discovery of WASP-8b, a transiting planet of $2.25 \pm 0.08 M_{\mathrm{Jup}}$ on a strongly inclined eccentric 8.15 -day orbit, moving in a retrograde direction to the rotation of its late-G host star. Evidence is found that the star is in a multiple stellar system with two other companions. The dynamical complexity of the system indicates that it may have experienced secular interactions such as the Kozai mechanism or a formation that differs from the "classical" disc-migration theory.
\end{abstract}

Key words. stars: individual: WASP-8 - techniques: photometric - techniques: spectroscopic - planet-star interactions planetary systems - techniques: radial velocities

\section{Introduction}

Transiting planets provide a wealth of information on the structure and formation of planets. The measurement of planet radius combined with its mass has found a surprising diversity in the mean densities and in particular "inflated" hot Jupiters. Spectroscopic measurement of the Rossiter-McLaughlin effect on the radial velocity during transits indicates that some of these planets may not be aligned with the rotation axes of their stars (see references in Winn 2010). The diversity in the observed spin-orbit misalignments is somewhat similar to that seen earlier in period and eccentricity distribution of planets detected by radial velocity surveys (see references in Udry \& Santos 2007, and references therein). The recent sharp rise in the detections of transiting planets is the outcome of successful ground-based wide transit searches surveys among which WASP (Pollacco et al. 2006) is the most prolific.

These discoveries have stimulated theoretical investigations of alternative formation scenarios to the migration theory (Lin et al. 1996; Wu \& Murray 2003). These alternative theories account for the discoveries of eccentric hot Jupiters on orbits not aligned with the rotation equator of their star (Wu \& Murray 2003; Fabrycky \& Winn 2009; Nagasawa et al. 2008; Barker \& Ogilvie 2009).

* Based on observations made with HARPS spectrograph on the 3.6-m ESO telescope and the EULER Swiss telescope at La Silla Observatory, Chile.

$\star \star$ Radial velocity data are only available in electronic form at the CDS via anonymous ftp to cdsarc.u-strasbg.fr (130.79.128.5) or via

http://cdsweb.u-strasbg.fr/cgi-bin/qcat?]/A+A/517/L1

\section{Observations}

\subsection{The WASP-8 multiple stellar system}

The star WASP-8 (TYC2 7522-505-1) at $\alpha(2000): 23^{\mathrm{h}} 59^{\mathrm{m}} 36.07^{\mathrm{s}}$, $\delta(2000):-35^{\circ} 1^{\prime} 52.9^{\prime \prime}$, was observed in 2006 and 2007 by the WASP-south telescope (Pollacco et al. 2006). It is a $V=$ 9.79 mag star with a Tycho $(B-V)$ color of 0.73 which is indicative of a G8 spectral type. The Infra-red Flux Method (IRFM) (Blackwell \& Shallis 1977), using GALEX, TYCHO-2, USNOB1.0 R-magnitude, and 2MASS broad-band photometry, yields a distance of $87 \pm 7 \mathrm{pc}$.

WASP-8 is identified in the CCDM catalogue (CCDM 23596-3502) as the A component of a system of three stars. The B component is a 15th magnitude red star, 4 arcsec south of A, and the third component $C$ is a 10th magnitude star (HIP 118299, HD 224664) 142 arcsec north of A. The radial velocity of HD 224664 is $4.7 \mathrm{~km} \mathrm{~s}^{-1}$ and stable over two years (Mayor, priv. com.) but differs from the WASP-8 value of $-1.5 \mathrm{~km} \mathrm{~s}^{-1}$. The proper motions of the components also differs. It is therefore unlikely that $\mathrm{C}$ and $\mathrm{A}$ are physically associated.

We measured the photometry and position of WASP-8 and its nearby star (B component) with the Euler CCD camera of the $1.2 \mathrm{~m}$ swiss Euler telescope at La Silla (see Fig. 1). By comparing with nearby stars, we obtained a magnitude difference $\Delta m_{V}=4.7, \Delta m_{I}=3.5$. A separation and a projected angle was measured on the deconvolved images (Gillon et al. 2007) and we obtained $4.83 \pm 0.01^{\prime \prime}$ and PA $=170.7 \pm 0.1^{\circ}$ (only internal errors being considered). Assuming that WASP- 8 and its B component are part of a multiple system, the color indices would represent those of an M star. A similar photometric analysis of the individual 2MASS archive images indicates that $\Delta m_{J}=2.7, \Delta m_{H}=2.2$, and $\Delta m_{K}=2.1$, which are also 



Fig. 1. Original (left) and deconvolved (right) $V$-band image from the Euler telescope of the A and B component of WASP-8.

indicative of an $\mathrm{M}$ star. The value mentioned in the Washington Visual Double Star Catalog measured 70 years ago indicates $4.0^{\prime \prime}$ and $\mathrm{PA}=170^{\circ}$ (Mason et al. 2001). This suggests little, if any, relative motion of the two stars over the 70-year time span between these observations. When compared with the proper motion in right ascension of WASP-8, about 100 mas/yr (Zacharias et al. 2004), this indicates a common proper motion pair. Given the distance of $W A S P-8$ the sky-projected separation of the pair is about 440 AU. Using available differential photometry, we estimate the temperature of the B component to be about $3700 \mathrm{~K}$.

\subsection{Photometric and radial-velocity observations}

WASP-8 was recorded simultaneously by two cameras of the WASP-south telescope during two seasons (2006 and 2007). Altogether 11224 independent photometric points were recorded with a typical sampling of $8 \mathrm{~min}$. Transit events were detected in data from the first observation season. This triggered radial velocity follow-up observations of WASP-8 in November 2007 with the Coralie spectrograph mounted at the Swiss Euler telescope (Baranne et al. 1996; Queloz et al. 2000; Pepe et al. 2002). With a combined analysis of the radialvelocity data and the photometry including additional WASP data from the 2007 season, a transit period of 8.15 days was found. No changes to the spectroscopic profile were detected, ruling out a blended eclipsing binary or starspots as the cause of the radial-velocity variation (Queloz et al. 2001) (see bottom diagram in Fig. 2). In the next season, observations with CORALIE were continued, revealing an additional drift in the $\gamma$ velocity of the system (Fig. 3). No second-order curvature term was detected.

On 25 August 2008, following up on the confirmation of the planet, a complete and densely sampled transit event was recorded in $R$ band with the Euler telescope to improve the determination of the transit parameters (Fig. 4). On 4 October 2008, a spectroscopic transit was measured with the HARPS spectrograph installed on the $3.6 \mathrm{~m}$ telescope at La Silla. During the sequence, 75 spectra (44 in the transit) were measured with an exposure time of $300 \mathrm{~s}$, corresponding to a typical signal-tonoise ratio per pixel of 50 . The radial velocity measurement from these spectra shows an obvious Rossiter-McLaughlin effect with a shape suggesting a non-coplanar orbit (Fig. 4). In addition, four spectra were measured on the same night before and after the transit to help us to determine the rate of change in the radial velocity outside the transit. Three measurements were obtained later at other phases of the system to improve the matching and zero point correction between CORALIE and HARPS data.
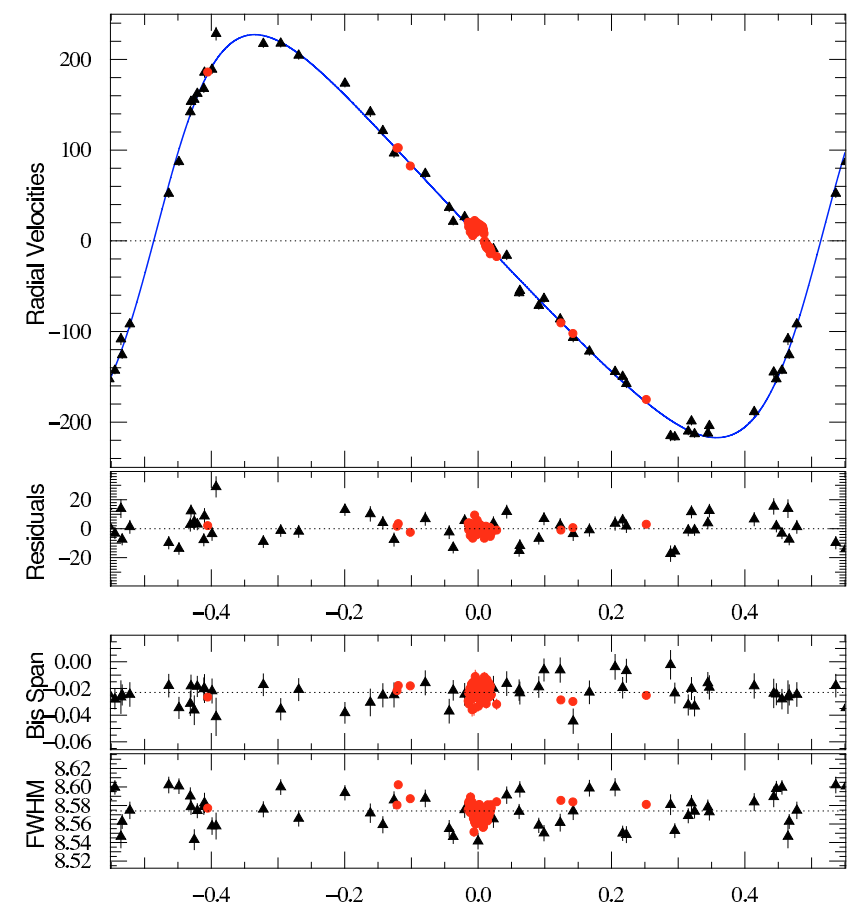

Fig. 2. Top: overall Keplerian fit to the RV data for Wasp-8b. Black triangles indicates Coralie data and red dots the Harps data. The long-term drift was removed to plot the velocity in phase (the zero is set at the time of the transit). Bottom: bissector span and FWHM (in km s${ }^{-1}$ unit) plotted with the phase of the orbit. The HARPS radial velocity data were shifted to correct from the $\gamma$ velocity difference with CORALIE.

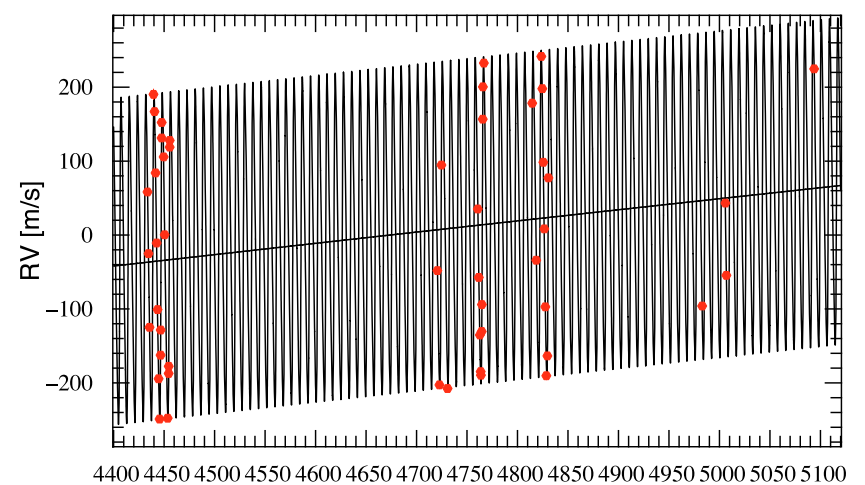

BJD - 2450000.0 [days]

Fig. 3. CORALIE radial velocity measurements (red dots) of WASP-8 superimposed on the best-fit solution (solid line).

During the measurement of the transit sequence, a significant change in telescope focus happened at JD 54744.592, improving the flux entering the fiber by a factor of 2 .

\section{Determination of system parameters}

\subsection{Spectral analysis}

The individual HARPS spectra were coadded and used for a detailed spectroscopic analysis of WASP-8. The results are displayed in Table 1. As in previous WASP-papers (Cameron et al. 2007), the analysis was performed using the UCLSYN spectral synthesis package and ATLAS9 models without convective overshooting with $H_{\alpha}$ and $H_{\beta} \mathrm{Na} \mathrm{I} \mathrm{D}$ and $\mathrm{Mg} \mathrm{I} \mathrm{b}$ lines as diagnostics for $T_{\text {eff }}$ and $(\log g)$. The abundances and the microturbulence 
D. Queloz et al.: WASP-8b: a retrograde transiting planet in a multiple system


Fig. 4. Top: radial velocity measurement phased with the transit (midtransit is at 0). Black triangles are CORALIE data and red dots HARPS data. Bottom: normalized transit photometry measurement of $W A S P-8$. Black triangles indicates SuperWASP data and red dots the $R$-band Euler photometry data. The best-fit model is superimposed in blue.

were determined in a similarly way to the work of Gillon et al. (2009) and used as additional $T_{\text {eff }}$ and $\log g$ diagnostics (Smalley 2005).

The Li I $6708 \AA$ line is detected in the spectra indicating an abundance of $\log \mathrm{A}(\mathrm{Li} / \mathrm{H})+12=1.5 \pm 0.1$, which implies an age of 3-5 Gyr according to Sestito \& Randich (2005). However, Israelian et al. (2009) noted that stars with planets have lower lithium abundances than normal solar-type stars, so the lithium abundance may not be a good age indicator for them.

The rotational broadening $v \sin i$ was measured by fitting the observed HARPS profiles of several unblended Fe I lines. A typical value of macroturbulence $v_{\text {mac }}=2 \mathrm{~km} \mathrm{~s}^{-1}$ was adopted and an instrumental profile determined from telluric absorption lines. We found that $v \sin i=2.0 \pm 0.6 \mathrm{~km} \mathrm{~s}^{-1}$, which is typical of a $\mathrm{G}$ dwarf of intermediate age.

\subsection{Analysis of the planetary system}

This whole data set was found to detect without doubt a planet transiting the star WASP-8. We analyzed together the photometric (WASP and Euler data) and the radial velocity data, including the spectroscopic transit sequence in this context. Our model was based on the transit modeling by Mandel \& Agol (2002) and the radial velocity description by Giménez (2006). The bestfit model parameters and their error bars were computed using a MCMC convergence scheme that solves all parameters together. For details of the code and fitting techniques, we refer to
Table 1. Stellar parameters of WASP-8 derived from spectroscopic analysis.

\begin{tabular}{ll|cc}
\hline \hline$T_{\text {eff }}$ & $5600 \pm 80 \mathrm{~K}$ & {$[\mathrm{Na} / \mathrm{H}]$} & $+0.22 \pm 0.07$ \\
$\log g$ & $4.5 \pm 0.1$ & {$[\mathrm{Mg} / \mathrm{H}]$} & $+0.21 \pm 0.04$ \\
$\xi_{\mathrm{t}}$ & $1.1 \pm 0.1 \mathrm{~km} \mathrm{~s}^{-1}$ & {$[\mathrm{Si} / \mathrm{H}]$} & $+0.29 \pm 0.09$ \\
$v \sin i$ & $2.0 \pm 0.6 \mathrm{~km} \mathrm{~s}^{-1}$ & {$[\mathrm{Ca} / \mathrm{H}]$} & $+0.24 \pm 0.12$ \\
{$[\mathrm{Fe} / \mathrm{H}]$} & $+0.17 \pm 0.07$ & {$[\mathrm{Sc} / \mathrm{H}]$} & $+0.23 \pm 0.05$ \\
$\log \mathrm{A}(\mathrm{Li} / \mathrm{H})+12$ & $1.5 \pm 0.1$ & {$[\mathrm{Ti} / \mathrm{H}]$} & $+0.24 \pm 0.08$ \\
& & {$[\mathrm{~V} / \mathrm{H}]$} & $+0.30 \pm 0.08$ \\
$\operatorname{dist}$ & $87 \pm 7 \mathrm{pc}$ & {$[\mathrm{Cr} / \mathrm{H}]$} & $+0.17 \pm 0.09$ \\
age & $3-5 \mathrm{Gyr}$ & {$[\mathrm{Co} / \mathrm{H}]$} & $+0.29 \pm 0.07$ \\
& & {$[\mathrm{Ni} / \mathrm{H}]$} & $+0.23 \pm 0.07$ \\
\hline
\end{tabular}

Notes. The quoted error estimates include those given by the uncertainties in $T_{\text {eff }}, \log g$, and $\xi_{\mathrm{t}}$, as well as atomic data uncertainties.

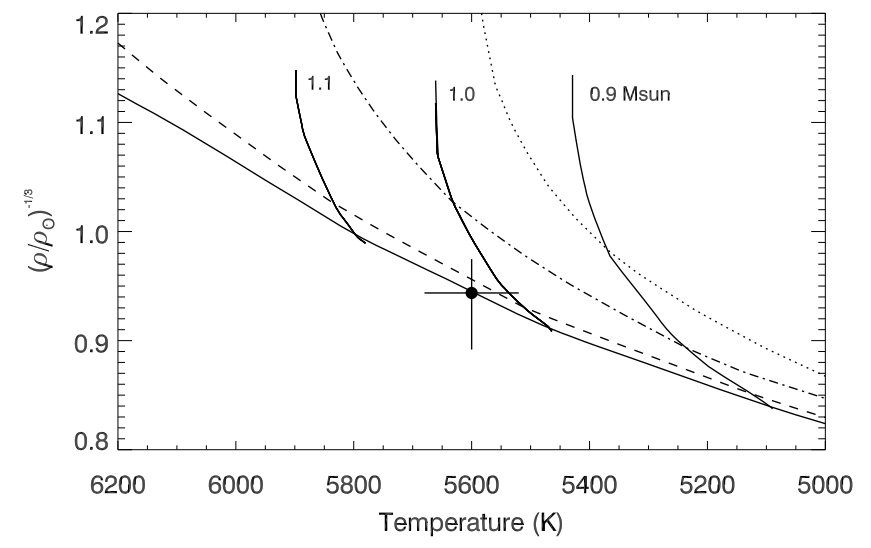

Fig. 5. Comparison of the best-fitting stellar parameters from the transit profile and spectroscopic analysis with evolutionary models interpolated at $[\mathrm{Fe} / \mathrm{H}]=0.17$. The isochrones are $100 \mathrm{Myr}, 1 \mathrm{Gyr}, 5 \mathrm{Gyr}$ and 10 Gyr. The evolutionary tracks are indicated for $0.9,1.0$ and $1.1 M_{\text {sun }}$.

Triaud et al. (2009); Collier Cameron et al. (2007). To obtain a coherent solution, we determined the mass of the star by comparing the spectroscopically-determined effective temperature and the stellar density outcome of the MCMC adjustment, with evolutionary tracks and isochrones of the observed metallicity from the stellar evolution model of Girardi et al. (2000). We converged iteratively on a stellar mass of $1.04(+0.02-0.09) M_{\odot}$ and an age younger than 6 Gyr (see in Fig. 5).

The free parameters of our model were the depth of transit $D$, the width of transit $W$, the impact parameter $b$, the period $P$, the epoch of transit centre $T_{0}$, the RV semi-amplitude $K, e \cos \omega$ and $e \sin \omega$ ( $e$ being the eccentricity and $\omega$ the angle of the periastron), and $V \sin I \cos \beta$ and $V \sin I \sin \beta$, with $V \sin I$ being the projection of the stellar equatorial rotation, and $\beta$ the projection of the angle between the stellar spin axis and the planetary orbit axis. In addition, we employed free normalization factors for each lightcurve (WASP and Euler) and each set of radial velocity ( $\gamma_{\mathrm{H}}$ for HARPS and $\gamma_{\mathrm{C}}$ for CORALIE), which enabled variations to be made in instrumental zero points. From these parameters, physical parameters were derived to characterise the planetary system. The best-fit set of parameters that minimize the $\chi_{\mathrm{r}}^{2}$ (reduced $\chi^{2}$ is 0.86) are listed in Table 2 as well as their related computed physical parameters. With this best-fit solution one computes for the CORALIE data $\chi^{2}=204$ with 48 measurements, and for HARPS data $\chi^{2}=188$ with 82 measurements which implies that additional jittering is present that is not accounted for by the fitted model. Since the main deviation is related to the CORALIE data, the uncertainties in the orbital 
Table 2. Fitted and physical parameters of the WASP-8 planetary system.

\begin{tabular}{lrl}
\hline \hline$D$ & 0.01276 & $(+0.00033-0.00030)$ \\
$W($ days $)$ & 0.1832 & $(+0.0030-0.0024)$ \\
$b\left(R_{\star}\right)$ & 0.604 & $(+0.043-0.040)$ \\
$P($ days $)$ & 8.158715 & $(+0.000016-0.000015)$ \\
$T_{0}(\mathrm{BJD}-2450000)$ & 4679.33394 & $(+0.00050-0.00043)$ \\
$K\left(\mathrm{~m} \mathrm{~s}^{-1}\right)$ & 222.23 & $(+0.84-0.60)$ \\
$\mathrm{d} \gamma / \mathrm{d} t\left(\mathrm{~m} \mathrm{~s}^{-1} \mathrm{yr}^{-1}\right)$ & 58.1 & $(+1.2-1.3)$ \\
$e \cos \omega$ & 0.02307 & $(+0.0010-0.0010)$ \\
$e \sin \omega$ & -0.3092 & $(+0.0024-0.0029)$ \\
$V \sin I \cos \beta$ & -0.873 & $(+0.059-0.064)$ \\
$\gamma_{\mathrm{C}}{ }^{a}\left(\mathrm{~m} \mathrm{~s}^{-1}\right)$ & -1565.76 & $(+0.16-0.21)$ \\
$\gamma_{\mathrm{H}}{ }^{-1}\left(\mathrm{~m} \mathrm{~s}^{-1}\right)$ & -1548.10 & $(+0.60-0.13)$ \\
$V \sin I \sin \beta$ & 1.59 & $(+0.08-0.09)$ \\
$R_{p} / R_{\star}$ & 0.1130 & $(+0.0015-0.0013)$ \\
$R_{\star} / a$ & 0.0549 & $(+0.0024-0.0024)$ \\
$\rho_{\star}\left(\rho_{\odot}\right)$ & 1.22 & $(+0.17-0.15)$ \\
$R_{\star}\left(R_{\odot}\right)$ & 0.945 & $(+0.051-0.036)$ \\
$M_{\star}\left(M_{\odot}\right)$ & 1.030 & $(+0.054-0.061)$ \\
$V \sin I\left(\mathrm{~km} \mathrm{~s}^{-1}\right)$ & 1.59 & $(+0.08-0.09)$ \\
$R_{p} / a$ & 0.00620 & $(+0.00036-0.00033)$ \\
$R_{p}\left(R_{J}\right)$ & 1.038 & $(+0.007-0.047)$ \\
$M_{p}\left(M_{J}\right)$ & 2.244 & $(+0.079-0.093)$ \\
$a(\mathrm{AU})$ & 0.0801 & $(+0.0014-0.0016)$ \\
$i\left(^{\circ}\right)$ & 88.55 & $(+0.15-0.17)$ \\
$e$ & 0.3100 & $(+0.0029-0.0024)$ \\
$\omega\left(^{\circ}\right)$ & -85.73 & $(+0.17-0.18)$ \\
$\beta\left(^{\circ}\right)$ & 123.3 & $(+4.4-3.4)$ \\
\hline
\end{tabular}

Notes. The error bars are calculated at $68 \%$ of the statistical distributions.

(a) Computed at JDB $=2454691.15781$.

solutions are most likely underestimated. However, the error bars in the Rossiter parameters are driven mostly by the HARPS ontransit data, and one can assume that they are almost correct.

Our best-fit solution corresponds to a giant planet with an eccentric $(e=0.3) 8.16$-day orbit and an additional long-term radial-velocity drift of $58 \mathrm{~m} \mathrm{~s}^{-1} \mathrm{yr}^{-1}$. The planet is dense with $2.25 M_{\mathrm{j}}$ and a radius of $1.04 R_{\mathrm{j}}$, in contrast to the substantial fraction of "inflated" hot Jupiters. Surprisingly, the projected angle between the orbital and stellar spin axes is found to be $\beta=123.3^{\circ}$, indicative of a retrograde orbit. We note that $V \sin I=1.59 \mathrm{~km} \mathrm{~s}^{-1}$ is in accordance with the line rotation broadening $v \sin i$ (in Table 1) derived by the spectral analysis.

We checked whether the partial defocusing of HARPS during the transit spectroscopic sequence had any effect on our result. We divided the series into two subsets and considered for each of them an independent offset $(\gamma)$. We obtain a solution with a marginal improvement in the $\chi^{2}$. By comparing the solution obtained from these two sets with that for the complete set, the angle $\beta$ was changed by $1.5 \sigma$. The defocusing problem does not affect the results of this paper.

\section{Discussion}

The detection of a hot Jupiter on an eccentric orbit that is misaligned with the stellar rotation axis and moving in a retrograde direction raises many questions about the origins of this system. Although the answer is beyond the scope of this paper, the visual faint companion and the drifting $\gamma$ velocity of the system are key components of the puzzle. From the observed separation between the $\mathrm{A}$ and $\mathrm{B}$ components, one can derive a most likely orbital semi-major axis $(a=1.35 \rho \approx 600 \mathrm{AU})$ (Duquennoy \& Mayor 1991). The observed radial-velocity drift is therefore unlikely to be related to the B component of the binary $\left(\dot{\gamma}<G M a^{-2}<1 \mathrm{~m} \mathrm{~s}^{-1} \mathrm{yr}^{-1}\right)$, suggesting that these is an additional closer companion of both unknown mass and period. The lack of curvature indicates that the companion is more massive than the transiting planet. This intermediate body is very likely to play a significant dynamical role in the system.

Apart from the complex dynamics of the whole system, the planet WASP- $8 b$ is a "standard" hot Jupiter. It orbits a metalrich star, which accounts for the observed increase in the incidences of hot Jupiters with the metallicity of the host star (Udry \& Santos 2007). The period of WASP- $8 b$ is longer than the 3-4 days typical value, but considering the eccentricity of its orbit, its periastron distance is typical of hot Jupiters.

The orbit misalignment of the planet with the stellar rotation axis of WASP- 8 is measured with the $\beta$ parameter. The true angle between the axes of the stellar and planetary orbits is usually called $\psi$ and is statistically related to $\beta$ through $\sin I$ (unknown) and the orbital inclination ( $i$ ) (see Fabrycky \& Winn 2009, for details). When $\beta$ deviates significantly from zero, this provides us with a lower limit to the $\psi$. When $\beta$ is beyond $90^{\circ}$, the orbital spin has the opposite direction to the stellar rotation provided that the orbit does not transit the star between its pole and its limb. According to Eq. (9) from Fabrycky \& Winn (2009), this condition is met when $I>3.6$ degree. By combining $V \sin I$, with the estimated age of the star, one can exclude such a small $I$ angle. Interpreted with the large $\beta$ value, we can conclude that a true retrograde orbit is the most likely scenario for WASP- $8 b$.

The origin of the unusual shape and orientation of the orbit of WASP- $8 b$ is possibly related to the Kozai mechanism (Kozai 1962; Wu \& Murray 2003) or the outcome of a violent dynamical interaction history. The evidence of two other bodies and a possible series of secular effects (Takeda et al. 2008) make the $W A S P-8$ system unique and interesting for additional dynamical studies and a test case for formation scenarios of hot Jupiters that constitute an alternative to the disc-migration mechanism.

\section{References}

Baranne, A., Queloz, D., Mayor, M., et al. 1996, A\&AS, 119, 373 Barker, A. J., \& Ogilvie, G. I. 2009, MNRAS, 395, 2268 Blackwell, D. E., \& Shallis, M. J. 1977, MNRAS, 180, 177 Cameron, A. C., Bouchy, F., Hébrard, G., et al. 2007, MNRAS, 375, 951 Collier Cameron, A., et al. 2007, MNRAS, 380, 1230 Duquennoy, A., \& Mayor, M. 1991, A\&A, 248, 485

Fabrycky, D. C., \& Winn, J. N. 2009, ApJ, 696, 1230

Gillon, M., Magain, P., Chantry, V., et al. 2007, ASP Conf. Ser., 366, 113 Gillon, M., Smalley, B., Hebb, L., et al. 2009, A\&A, 496, 259

Giménez, A. 2006, ApJ, 650, 408

Girardi, L., Bressan, A., Bertelli, G., \& Chiosi, C. 2000, A\&AS, 141, 371 Israelian, G., Delgado Mena, E., Santos, N. C., et al. 2009, Nature, 462, 189 Kozai, Y. 1962, AJ, 67, 591

Lin, D. N. C., Bodenheimer, P., \& Richardson, D. C. 1996, Nature, 380, 606 Mandel, K., \& Agol, E. 2002, ApJ, 580, L171

Mason, B. D., Wycoff, G. L., Hartkopf, W. I., et al. 2001, AJ, 122, 3466 Nagasawa, M., Ida, S., \& Bessho, T. 2008, ApJ, 678, 498

Pepe, F., Mayor, M., Galland, F., et al. 2002, A\&A, 388, 632

Pollacco, D. L., Skillen, I., Cameron, A. C., et al. 2006, PASP, 118, 1407

Queloz, D., Mayor, M., Weber, L., et al. 2000, A\&A, 354, 99

Queloz, D., Henry, G. W., Sivan, J. P., et al. 2001, A\&A, 379, 279

Smalley, B. 2005, Mem. Soc. Astron. It. Suppl., 8, 130

Sestito, P., \& Randich, S. 2005, A\&A, 442, 615

Takeda, G., Kita, R., \& Rasio, F. A. 2008, ApJ, 683, 1063

Triaud, A. H. M. J., Queloz, D., Bouchy, F., et al. 2009, A\&A, 506, 377

Udry, S., \& Santos, N. C. 2007, ARA\&A, 45, 397

Winn, J. N. 2010 [arXiv: 1001.2010]

Wu, Y., \& Murray, N. 2003, ApJ, 589, 605

Zacharias, N., Monet, D. G., Levine, S. E., et al. 2004, BAAS, 36, 1418 